\title{
Surgical treatment of displaced intra-articular calcaneal fracture using a single small lateral approach
}

\author{
Mohamed F. Mostafa $\cdot$ Gamal El-Adl • \\ Ehab Y. Hassanin $\cdot$ M-Serry Abdellatif
}

Received: 1 February 2009/Accepted: 6 February 2010/Published online: 9 March 2010

(C) Springer-Verlag 2010

\begin{abstract}
The objective of this study was to evaluate the outcome of semi-open reduction and minimal internal fixation through a single small lateral approach as a minimally invasive technique for treatment of displaced intraarticular calcaneal fractures. This prospective study was conducted on eighteen patients (16 men and 2 women). The average age was 37.7 (22-55). The most common cause of injury was a fall from height in fourteen patients. Patients were operated on within a mean time of 4.8 days of admission (1-11 days) and were followed up for an average period of 24.1 months (6-39 months). Patients were evaluated clinically using the Creighton-Nebraska Heath Foundation Assessment score of Crosby and Fitzgibbons (J Bone Joint Surg (Am) 72-A:852-859, 1990). The scoring system proposed by Knirk and Jupiter was used for radiological assessment of the posterior subtalar joint (Knirk and Jupiter in J Bone Joint Surg (Am) 68-A: 647-659, 1986). The skin incision healed in all cases without necrosis, infection, or sural nerve injury. All fractures healed after an average of 8 weeks (7-10 weeks), and patients returned to the routine daily activities after an average time of 4.3 months (3-7 months). In conclusion, semi-open reduction and minimal internal fixation through a small lateral approach is an effective treatment for carefully selected cases of displaced intra-articular calcaneal fractures.
\end{abstract}

M. F. Mostafa ( $\square)$ · G. El-Adl · E. Y. Hassanin .

M.-S. Abdellatif

Department of Orthopedic Surgery and Traumatology,

Faculty of Medicine, Mansoura University,

PO Box 2, Mansoura 35516, Egypt

e-mail: thabetortho20032003@yahoo.com
Keywords Foot injuries - Fracture fixation - Internal · Minimally invasive surgical procedures

\section{Introduction}

Calcaneus fractures are the most common fracture of the tarsal bones, yet controversy still exists on the best treatment for these disabling injuries $[2,9,15,16,20]$. However, as a better understanding of fracture patterns with computed tomography scans and modern surgical techniques and hardware has improved outcomes and lowered morbidity, a trend has developed toward open reduction and internal fixation (ORIF) for displaced, intra-articular calcaneus fractures $[2,4,6,9,16,27]$.

Calcaneus fractures often results in a varus deformity with heel widening, loss of calcaneal height, and subtalar joint incongruency. ORIF can be used to address deformities, restoring the anatomic morphology of the calcaneus, and thereby the biomechanics and function of the hindfoot. Restoring heel width prevents chronic peroneal tendenitis secondary to impingement from lateral wall blowout of the calcaneus, and restoring the length and alignment of the Achilles tendon maintains plantar flexion strength $[16,20,27]$. ORIF also provides the opportunity for anatomic reduction and rigid internal fixation of the subtalar joint. Normal subtalar motion is integral for the foot to adapt on uneven surfaces with inversion and eversion $[6,20,29]$.

The extensile lateral approach has gained wide popularity for the surgical fixation of the intra-articular calcaneal fracture [3]. It provides excellent exposure, allowing access to manipulate and fix the fracture fragments [28]. Results from the extensile lateral approach can be rewarding, but soft tissue complications can be serious. 
This applies particularly to open calcaneal fractures, smokers, or patients with diabetes $[1,12]$. For this reason, there has been renewed interest in small-incision surgery for calcaneal fractures. This approach is not new. In 1982, McReynolds [18] popularized the medial approach, with the results published by Burdeaux [5]. In 1983, in a preliminary report, Stephenson [26] described a combined lateral and medial approach for the treatment of displaced intra-articular calcaneus fractures.

The purpose of the current study is to evaluate the results of open reduction through a small lateral approach with percutaneous fixation as a minimally invasive surgical treatment of the displaced intra-articular calcaneal fracture.

\section{Patients and methods}

From May 2005 to July 2008, eighteen displaced intraarticular calcaneus fractures in eighteen patients were managed surgically with open reduction through small lateral incision with minimal internal fixation. The indication for surgical treatment was more than two millimeters displacement of the subtalar joint, with a decrease in the Bohler's angle, incongruity of the articular surfaces of the posterior facet, and widening of the heel.

Among the eighteen patients included in this study, 16 were men and 2 were women. Their average age was 37.7(22-55). The right side was involved in 12 cases and the left side in 6 cases. The mechanism of injury was a fall from height in fourteen patients and a motor-vehicle accident in four. All cases were closed fractures. Most of the young adult patients were smokers (but not heavy) and were instructed to stop smoking until complete wound healing. Only one patient was diabetic and this was controlled. Three patients had associated injuries.

At the time of admission to the hospital, anteroposterior, lateral, axial, and internal oblique radiographs of the fractured calcaneus and oblique radiographs of the injured foot as well as lateral and axial radiographs of the normal calcaneus were taken. A pre-operative CT scan was taken for all cases to obtain better appreciation of the size of the displaced fracture fragments and the number of fracture lines that had to be identified and surgically reduced, with special reference to the anterolateral fragment.

From the radiographs and CT scan, the type of fracture was determined, and the pre-operative tuber-joint angle, and calcaneal height and width were measured. Fracture classification was based on the method described by Sanders 1993 which relies on sagittal reconstruction of CT images, reformatted parallel and perpendicular to the posterior facet of the subtalar joint [23]. An addition to the Sanders classification was used to include the anterior secondary fracture lines and the degree of comminution (Fig. 1). Absence of an anterior secondary fracture subcategorized as 0 . The presence of one anterior fracture line that extended either into the plantar surface of the
Fig. 1 a Diagram of lateral aspect of calcaneus.

Line 1 represents the primary shear fracture; line 2 represents the secondary compression fracture giving the tongue fragment; line 3 represents the secondary compression fracture of the joint depression type. When there is absence of an anterior secondary fracture, the subtype is 0 ; $\mathbf{b}$ and $\mathbf{c}$ The presence of one fracture line extending to either the calcaneocuboid joint or the plantar surface proximal to the joint is subtype 1 . d The presence of more than one anterior fracture is subtype 2 . e Extensive comminution is subtype 3
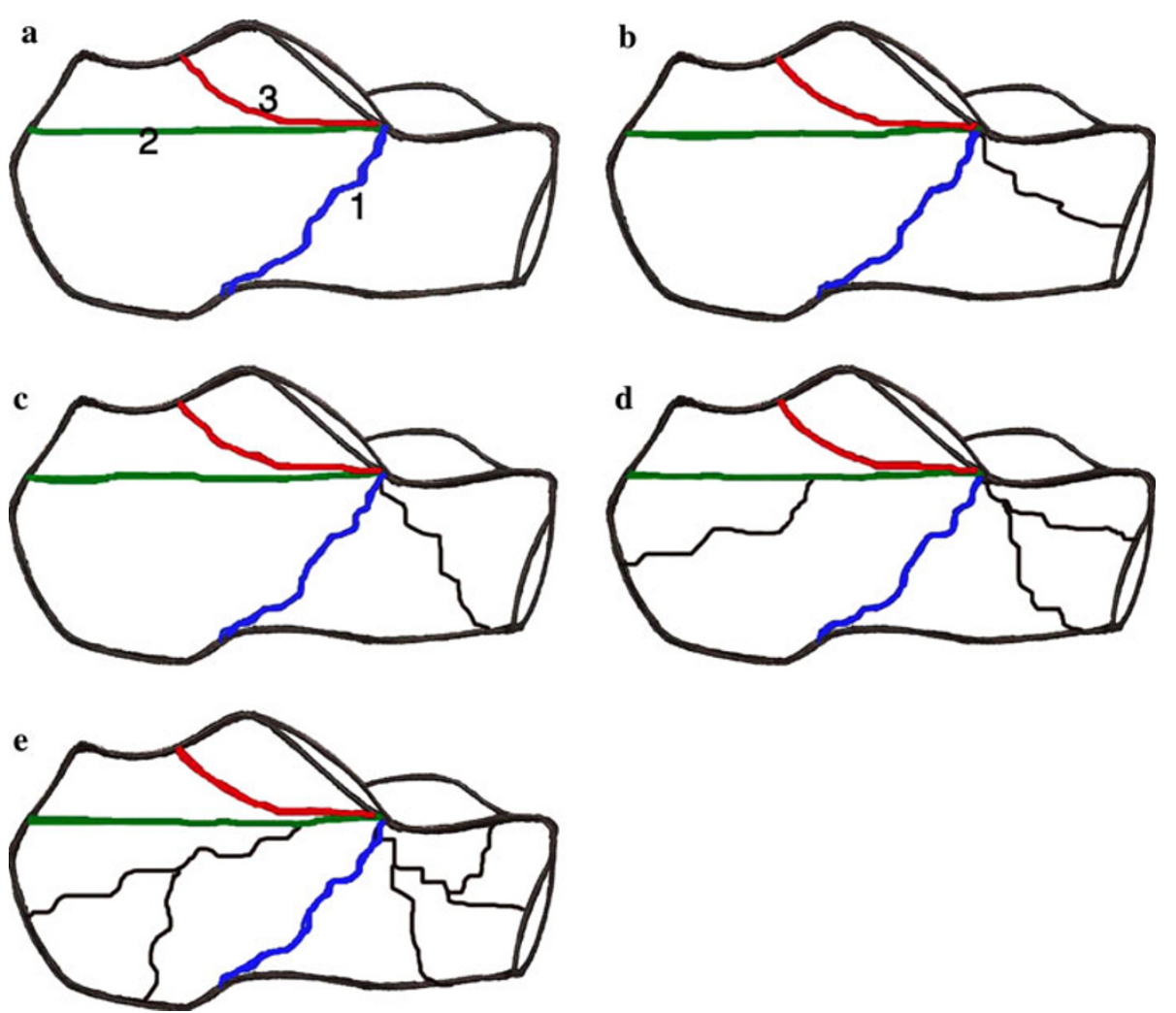
calcaneus just proximal to the calcaneocuboid joint or into the calcaneocuboid joint was subcategorized as 1 . Moderate comminution of the fracture, as defined as the presence of more than one anterior and one posterior secondary fracture line on the lateral radiograph, was subcategorized as 2 . An extensively comminuted fracture that could not be easily classified due to the numerous fracture lines and major displacement of the fragments was subcategorized as 3. Any of the Sanders type II or Type III fractures can be joint depression types or tongue types according to Essex-Lopresti.

None of the eighteen cases included in this study were two-part shear fractures (type I). There were twelve cases of type II fractures; 3 type IIA0, 3 type IIB0, one type IIC0, four type IIB1, and one type II C1. Six cases were type III fractures; one type IIIAB0, one type IIIAB1, one type IIIAB2, one type IIIBC 0 , one type IIIBC1, and one type IIIAC0. Twelve cases were classified as a joint depression type and six as tongue depression type. Surgery was performed after an average of duration of 4.83 days from admission (range 1-11 days).

\section{Surgical technique}

At the time of admission to the hospital, the patient's foot was placed in a Jones pressure dressing, and a posterior plaster splint applied. The extremity was elevated, and ice applied in an effort to minimize swelling and avoid blisters. In operating room, the patient was placed in a semi-lateral position with a sandbag under the ipsilateral buttock and the fractured heel uppermost. A thigh tourniquet was applied, and the foot rested on a wrap of towels that, at varying times, allowed the foot to hang freely without support once the towels were moved proximal to the ankle joint (Fig. 2a). Care was taken to place pads under the peroneal, hip, and axillary regions of the dependent side and to place a pillow between the legs.

The small lateral approach, which has been used at the Campbell clinic [19] and was described by Carr [7] as a limited sinus tarsi approach, was done for all cases. An incision approximately five to six centimeters in length was made laterally. It begins from the anterolateral corner of the calcaneocuboid joint and extends posteriorly in almost a straight line, with the foot held in $90^{\circ}$ to the ankle and ends $1-2 \mathrm{~cm}$ anterior to the tendocalcaneus (Fig. 2b). Using sharp dissection, this incision was carried down to the sheath of the peroneal tendons. Once the sural nerve and the short saphenous vein were identified and retracted, the peroneal sheath was opened along its anterior border where it meets the stem of the inferior extensor retinaculum to expose the anterolateral border of the sinus tarsi. The extensor digitorum brevis distally is elevated only to the extent enough to expose the calcaneocuboid joint. The
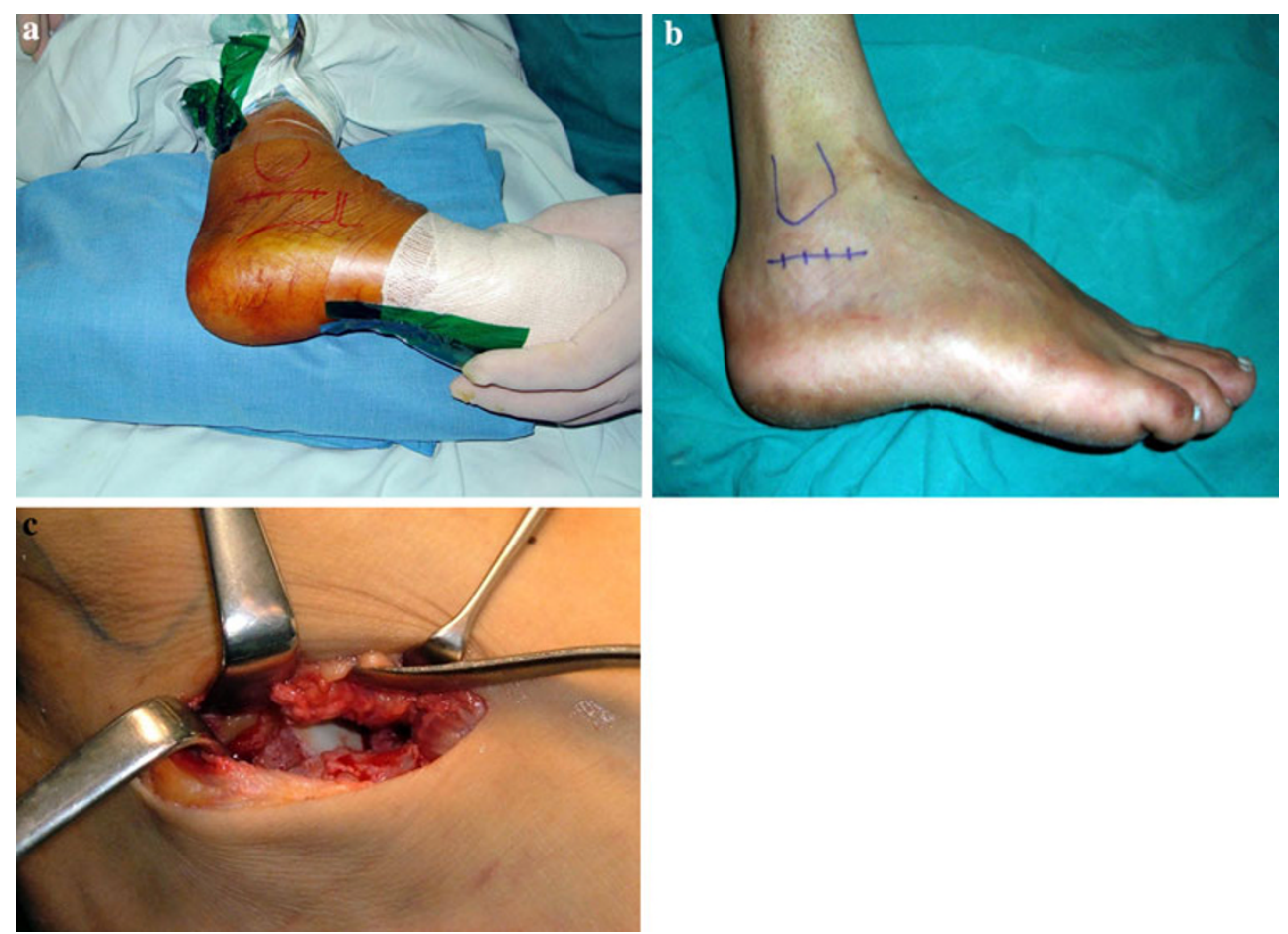

Fig. 2 a Photograph of a patient in the lateral position with the affected limb uppermost and a wrap of towels underneath the ankle; b the planned small lateral incision; c the depressed portion of the posterior facet 
cervical ligament was left intact, and the sinus tarsi dissected to the extent necessary to expose the anterior aspect of the posterior calcaneal facet.

The lateral wall of the calcaneus was then exposed by sharp dissection with a thin osteotome or periosteal elevator by first dissecting plantarward beneath the sheath of peroneal tendons, and then posteriorly where the distal end of calcaneofibular ligament and the lateral talocalcaneal ligament are identified and excised en-bloc. These ligaments are retracted along with the sheath of peroneal tendons which forms the posterior section of the capsule of the subtalar joint [23]. The lateral wall was sometimes surprisingly thin and comminuted. Therefore, it was possible to keep the lateral wall fragments together and attached to overlying tissue, and avoid separation by dissection, and retract it like a door to expose the depressed fracture fragment (Fig. 2c). In cases of tongue-type fractures, it was possible to continue dissection bluntly by finger tip or blunt periosteal elevator along the superior aspect of the calcaneal tuberosity to expose the posterior portion of posterior facet. With the foot held in valgus and some degree of equinus, the lateral wall and the peroneal sheath are pulled with a small stout-toothed retractor. After sharp dissection of any remaining capsular tissue and washing out the fracture hematoma, it was possible to scout the displaced posterior articular facet. If inspection was difficult, an Inge retractor was placed into the depths of the sinus tarsi, pressing up on the neck of the talus and down on the sinus tarsi portion of the calcaneus to give a better view as far as the sustentaculum tali.

At this time, the fracture was reduced by inserting a narrow Langenbeck elevator or 5-6 $\mathrm{mm}$ osteotome deep to the depressed fragment and elevating it into a proper position both medially and laterally. In type III injuries, with a second sagittal fracture line through the posterior facet medial to the first fracture, care was taken to elevate both fragments. The posterior facet of the talus was used as a template by pushing the depressed fragment against it into proper alignment with the supero-medial fragment. Temporary fixation was performed with two Kirschner wires (K-wires) ranging in size from 1.5 to $2 \mathrm{~mm}$ according to the size of the displaced fragment. The wires were driven through the subchondral bone from lateral to medial perpendicular to the fracture. One wire was used as a joystick to hold the fragment reduced, while the other wire driven across the fracture. This was technically easy for joint depression type fractures but, for tongue types, the posterior end of the tongue fragment had to be held in a reduced position with the tuberosity fragment using a large AO bone-reduction forceps. Since the posterior facet of calcaneus is convex upward, it was difficult to visualize the most medial portion of the facet from a small lateral approach. However, after reduction and temporary fixation, the posterior subtalar joint was assessed for congruity and accuracy of reduction through intra-operative Broden's views.

Upon congruent reduction of the posterior facet, the Kirschner wires were replaced sequentially with $4-\mathrm{mm}$ cancellous screws over washers using the lag effect. When elevation of the depressed fragment left a defect in cancellous bone, autogenous cortico-cancellous bone graft taken from the ipsilateral anterior iliac crest or synthetic bone substitutes (ceraform; calcium phosphate hydroxyapatite $65 \%$ and tricalcium phosphate $35 \%$, Teknimed S.A. France) was used not only to pack the defect but also to support the elevated posterior facet.

Attention was next turned to realigning the tuberosity fragment. In the tongue-type fracture, the superomedial fragment is longer and extends more posteriorly than it does in the joint depression type fracture [5, 10]. Therefore, greater manipulation of the tuberosity fragment was done in tongue depression type fractures than for the joint depression types. Guided by fluoroscopy, two 2- or 3-mm Kirschner wires were inserted on either side of Achilles tendon through the superior aspect of the posterior calcaneus. The wires were directed to exit the fracture just below the inferior margin of the anterior portion of the displaced posterior facet. The wires should not be driven in beyond the facet. An assistant places a thumb under the base of the heel at the level of the fracture, with one hand on the lateral wire and the other hand on the forefoot. The thumbs act as a fulcrum to force the forefoot plantigrade and to pull the tuberosity down. While the tuberosity was held in a reduced position using large AO bone-reducing forceps (one limb of the forceps laterally behind the peroneal tendons and the other medially behind the neurovascular bundle), a medial $\mathrm{K}$-wire was inserted into the superomedial fragment to hold the reduced medial wall. Axial views of the calcaneus were obtained to check the reduction of medial wall, alignment of the tuberosity, and direction of medial K-wire. The lateral K-wire may be bent during the reduction maneuver and can be replaced with another K-wire; this is inserted into the anterior process of the calcaneus to stabilize the tuberosity with the anterior portion and to support the posterior facet. If the anterior fracture line extends to the calcaneocuboid joint, transverse lag screws were used to fix fractures of the anterior process. The lateral K-wire could then be driven across the calcaneocuboid joint to gain purchase in the cuboid (Fig. 3). Anteroposterior and oblique radiographs were obtained to confirm that the wires were directed toward the calcaneocuboid joint and had not strayed medially or laterally. Finally, the lateral wall is impacted by squeezing the heel to reduce the calcaneal width.

Before closure of the wound, the tourniquet was released, and hemostasis obtained. If blood continued to 

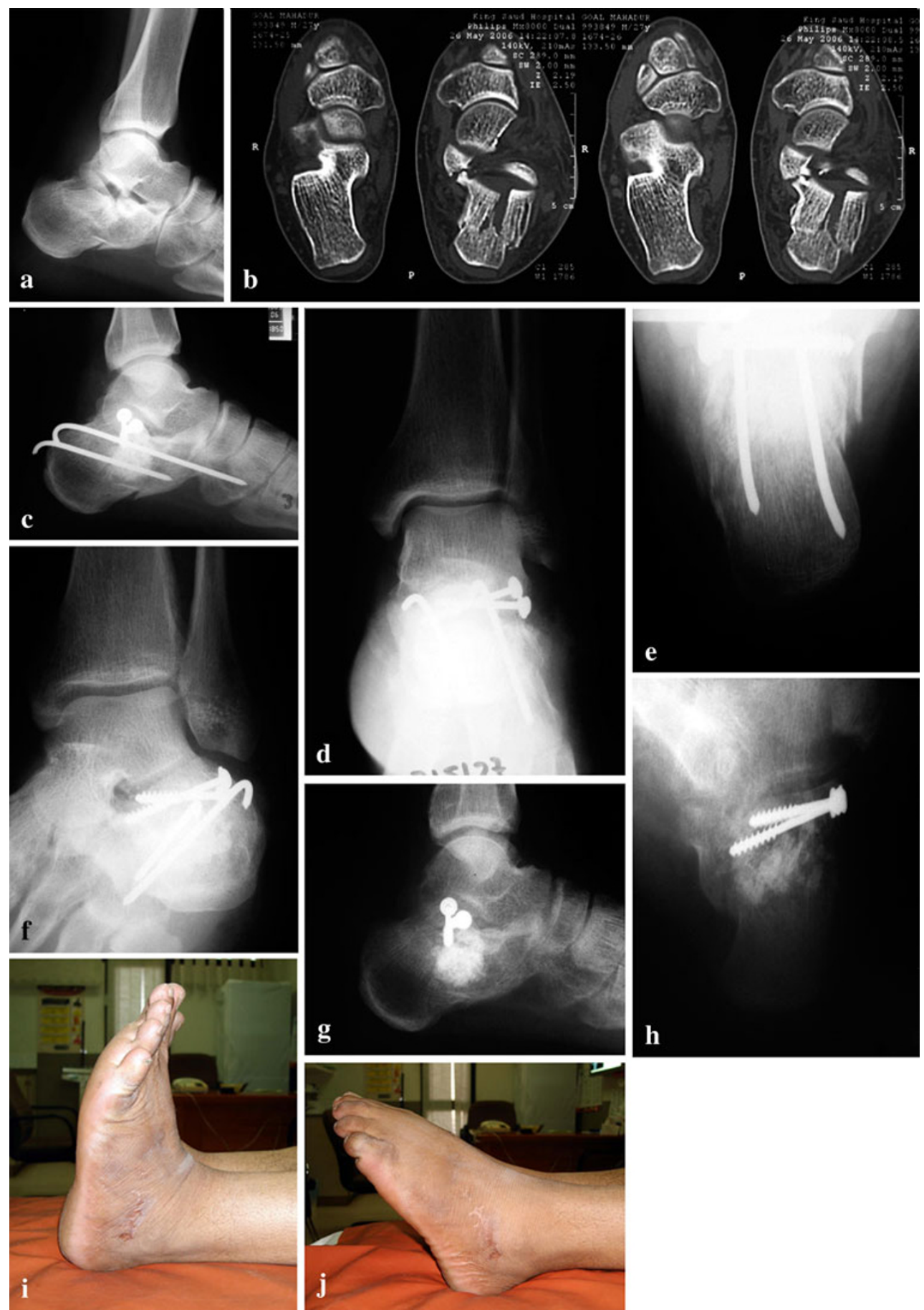

Fig. 3 a A 27-year-old man sustained a fall from height and had a joint depression fracture type IIIB0; $\mathbf{b}$ the CT scan revealed orientation of fracture lines and degree of displacement; $\mathbf{c}, \mathbf{d}, \mathbf{e}$, and f the postoperative lateral, anteroposterior, axial, and internal oblique

ooze from bone, the wound was closed over a small drain, a bulky dressing was applied, and the extremity elevated. No plaster splint or cast was required except in two patients in whom the fracture was comminuted and fixation of the tuberosity to anterior process insecure. As soon as the radiographs. One K-wire was advanced to the cuboid to add more stability to the tuberosity fragment, and synthetic bone substitute was used to fill the defect; $\mathbf{g}$ and $\mathbf{h}$ Follow-up radiographs after removal of the K-wire; $\mathbf{i}$ and $\mathbf{j}$ satisfactory functional results

patient was comfortable and the wound appeared to be satisfactory, exercises for subtalar motion were begun. These were usually be started 7-10 days postoperatively and included assisted active and active circumduction, inversion, and eversion movements of heel under the 
supervision of a physiotherapist in an effort to re-establish motion of the subtalar joint.

Sutures were removed after 3 weeks. At 4-6 weeks after surgery, gentle strengthening exercises for the muscles controlling the foot and ankle were started. Partial weightbearing was allowed using crutches when the fracture had healed radiographically and after removal of Kirschner wires, usually by $8-10$ weeks. Thereafter, full weightbearing without support was gradually resumed over a period of 4-6 weeks.

The mean duration of follow-up was 24.1 months (range 6-39 months). Patients were evaluated clinically using the Creighton-Nebraska Health Foundation Assessment score of Crosby and Fitzgibbons [8] (Table 1). Measurement of the range of movement of the subtalar joint was taken by a standard goniometer. The special pointer of McMaster [17], a gravity goniometer or the special jig of Leung [14], was not available during this study. The swelling of the heel was judged mild, moderate, or severe compared with the normal side. Radiological evaluation was made by obtaining serial radiographs of the affected calcaneus (lateral, axial, and internal oblique views) at follow-up. Bohler's angle and calcaneal height were measured from the lateral radiographs, and calcaneal width measured from the axial films. The results were compared with the measures obtained from the normal calcaneus obtained earlier in this study. The criteria used by Knirk and Jupiter 1986 [13] and adopted by Leung [14] were used to assess articular incongruity and degree of arthritis of posterior subtalar joint (Table 2).

Data were analyzed using SPSS for windows (version 8.0). The Pearson Chi-Square test, independent sample $t$ test, and one-way ANOVA test were used to define relations between clinical, radiological, and functional results. Statistical significance was set at $P<0.05$.

\section{Results}

The small lateral approach selected for this study was adequate for exposure of the posterior facet, allowing placement of bone graft, and reduction of the lateral wall. Indirect reduction of the medial wall was amenable by closed manipulation of the tuberosity fragment. Use of bone graft was not routine but needed in eleven cases (autogenous iliac bone in 7 cases and synthetic bone substitute in 4 cases). The skin incision healed well in all cases despite early mobilization without necrosis, infection or sural nerve damage. The Kirschner wires were removed after a mean time of 8.9 weeks (range, 8-10 weeks). The fracture had healed in all cases after an average time of 8 weeks (7-10 weeks), and all patients returned to their usual activity after a mean time of 4.3 months (range 3-7 months).
Table 1 Creighton-Nebraska Health Foundation assessment sheet for fractures of the calcaneus

\begin{tabular}{|c|c|}
\hline Item & Points* \\
\hline \multicolumn{2}{|l|}{ Pain (30 points) } \\
\hline \multicolumn{2}{|l|}{ Activity } \\
\hline No pain when walking or ignores pain & 15 \\
\hline Mild pain when walking; takes aspirin & 10 \\
\hline Moderate pain when walking; takes codeine & 5 \\
\hline Severe pain when walking; severe limitations & 0 \\
\hline \multicolumn{2}{|l|}{ Rest } \\
\hline No pain at rest or ignores pain & 15 \\
\hline Mild pain at rest & 10 \\
\hline Moderate pain at rest & 5 \\
\hline Severe pain at rest & 0 \\
\hline \multicolumn{2}{|l|}{ Activity (20 points) } \\
\hline Unlimited walking and standing & 20 \\
\hline $\begin{array}{l}\text { Walks } 5-10 \text { blocks; stands intermittently } \\
\text { for more than half an hour }\end{array}$ & 15 \\
\hline Walks $1-5$ blocks; stands half an hour or less & 10 \\
\hline Walks less than 1 block (indoor only) & 5 \\
\hline Can not walk & 0 \\
\hline \multicolumn{2}{|l|}{ Range of inversion/eversion (20 points) } \\
\hline $25^{\circ}-20^{\circ}=80-100 \%$ & 20 \\
\hline $20^{\circ}-15^{\circ}=60-80 \%$ & 15 \\
\hline $15^{\circ}-10^{\circ}=40-60 \%$ & 10 \\
\hline $10^{\circ}-5^{\circ}=20-40 \%$ & 5 \\
\hline $5^{\circ}-0^{\circ}=0-20 \%$ & 0 \\
\hline \multicolumn{2}{|l|}{ Return to work (20 points) } \\
\hline Full time, same job & 20 \\
\hline Full time, with restrictions & 15 \\
\hline Full time, change job & 10 \\
\hline Part time with restrictions & 5 \\
\hline Can not work & 0 \\
\hline \multicolumn{2}{|l|}{ Change in shoe size ( 5 points) } \\
\hline No change & 5 \\
\hline Change & 0 \\
\hline \multicolumn{2}{|l|}{ Swelling (5 points) } \\
\hline None & 5 \\
\hline Mild & 3 \\
\hline Moderate & 2 \\
\hline Severe & 0 \\
\hline
\end{tabular}

* 90-100 points is an excellent result; $80-89$ a good result; $65-79$ a fair result; and 64 or less a poor result

\section{Clinical results}

There were five excellent $(27.8 \%)$, nine good $(50.0 \%)$, two fair $(11.1 \%)$, and two poor $(11.1 \%)$. For simplicity, the excellent and good cases were rated satisfactory $(77.8 \%)$, and the fair and poor cases were rated unsatisfactory $(22.2 \%)$. Pain at the lateral aspect of heel was the main 
Table 2 Scoring system for posterior subtalar joint

\begin{tabular}{lll}
\hline Grade & $\begin{array}{l}\text { Articular incongruity } \\
\text { (mm step-off) }\end{array}$ & Arthritic changes \\
\hline 0 & $0-1$ & None \\
1 & $1-2$ & $\begin{array}{l}\text { Slight joint-space narrowing } \\
\text { Marked joint-space narrowing, } \\
\text { osteophyte formation } \\
2\end{array}$ \\
$2-3$ & $\begin{array}{c}\text { Bone-on-bone, osteophyte } \\
\text { formation, cyst formation }\end{array}$ \\
3 & $>3$ & \\
\hline
\end{tabular}

complain of the majority of patients. Thirteen patients (72.3\%) developed mild pain on walking, and only five patients $(27.8 \%)$ showed no pain at rest or on activity. Pain was tolerable and developed only after long walk in ten patients, while the remaining three patients required mild analgesics to relief pain at rest. Pain was related to subtalar joint incongruity in five patients, and peroneal tendons irritation in eight patients. No pain developed related to irritation by implant used. No cases of subluxation of peroneal tendons could be detected on follow-up. This may be attributed to preservation of the superior peroneal retinacula in most of cases. Thirteen patients $(72.3 \%)$ could walk and stand for unlimited time despite the presence of pain in eight of them. The remaining five patients $(27.8 \%)$ could walk for 5-10 blocks and stand intermittently for more than half an hour. Only three patients (16.7\%) showed a near normal range of motion of the subtalar joint with an average range of inversion to eversion movement of $96 \%$ from normal (range, 95-98\%), while the majority of patients $(72.3 \%)$ showed an average range of motion of $72.2 \%$ from normal (range, $63-80 \%$ ).

Fourteen patients $(77.8 \%)$ returned to their previous occupation full time, while three patients (16.7\%) attended the same job but with some restriction, and only one patient changed his job to a more sedentary one. This patient had a type IIIAB2 joint depression fracture, associated with other injuries, had a preoperative Bohler's angle of $-20^{\circ}$, and had the lowest score for functional results. Swelling of the heel was a common subjective and objective finding which was mostly soft tissue in origin and took a long time to resolve. Out of the eighteen patients included in this study, eleven patients $(61.1 \%)$ had mild swelling and six patients $(33.3 \%)$ moderate swelling.

\section{Radiological results}

The average preoperative Bohler's angle was $5.1^{\circ}$ (range, $-20^{\circ}$ to $19^{\circ}$ ), and the average angle at follow-up was $34.6^{\circ}$ (range, $20^{\circ}-45^{\circ}$ ). This change represented an average restoration of Bohler's angle to $91.4 \%$ of normal (range, $67-100 \%)$. Similarly, calcaneal height showed an average correction to $95.2 \%$ of normal height (range, $85-100 \%$ ).
The average residual widening of the calcaneus at followup was $9.2 \%$ of normal (range, 3-27\%). The majority of patients $(72.2 \%)$ had a congruent and non-arthritic joint, three patients $(16.7 \%)$ had $1-2-\mathrm{mm}$ step of the articular surface of posterior facet and slight joint narrowing, and only two patients (11.1\%) showed 2-3-mm step and significant arthritic changes.

We were unable to detect a significant relation between age, gender, mechanism of trauma, presence or absence of associated injuries, type of fracture, or the use of graft and the final functional result; this may be due to the small number in our sample. Nevertheless, the majority of patients $(91.6 \%)$ with a type II fracture had satisfactory results (excellent and good).

Patients with satisfactory functional results had a mean correction of Bohler's angle by $93.1 \%$ compared to those with unsatisfactory results where correction averaged $85.3 \%$ $(P=0.12)$. Patients with satisfactory results also had a mean correction of $96.6 \%$ in calcaneal height, significantly more than the patients with unsatisfactory results $(90.0 \%$, $P=0.001)$. The mean percentage correction in Bohler's angle was significantly higher in patients could walk and stand without limitation (95.2\%) than those who could walk 5-10 blocks and stand intermittently for more than half an hour $(81.6 \%, P=0.001)$. Patients who could walk and stand without limitation also had a mean percent correction of calcaneal height (96.3\%) significantly higher than those who could walk for 5-10 blocks and stand intermittently for more than half an hour $(91.2 \%, P=0.004)$.

The mean percentage residual calcaneal widening in patients with unsatisfactory functional results $(11.3 \%)$ was more than the patients with satisfactory results $(8.6 \%$, $P=0.5$ ). The mean percentage residual thickening in patients with moderate swelling $(7.2 \%)$ and in patients who had to change their shoe size $(12.7 \%)$ was not higher than those with no swelling $(3 \%, P=0.1)$ and in patients who did not change shoe size $(8.5 \%, P=0.3)$.

There was a significant relation between the identification of a congruent non-arthritic subtalar joint and the presence of satisfactory functional results $(P=0.01)$. All patients with a congruent non-arthritic subtalar joint developed a range of inversion to eversion significantly greater than $60 \%$ of normal range $(P=0.001)$. Patients with greater joint congruity and without arthritic changes had greater ability to walk and stand for unlimited periods $(P=0.01)$. All patients with a congruent non-arthritic subtalar joint returned to their previous occupation $(P=0.001)$.

\section{Discussion}

The prognosis for an extra-articular calcaneal fracture is uniformly good unlike that for intra-articular types [22]. 
The treatment of these fractures remains controversial partly because of problems with different classification systems, indications for operative treatment, and different assessments of the clinical and radiological results. The recent increase in operative treatment for displaced intraarticular fractures has added to the understanding of these difficult fractures $[8,26]$. A biomechanical study of Perry [21] has confirmed the important role of the subtalar joint in relieving the ankle from rotational forces during walking. Without this relieving mechanism, the ankle may develop secondary degenerative arthritis. Fractures of the calcaneus with involvement of the subtalar joint are actually split-depressed fractures analogous to displaced fractures of the tibial plateau. Such fractures should be treated like any other intra-articular fractures by anatomical reduction, absolute stable fixation, and early mobilization.

Stephenson [26] used a combined medial and lateral approach and found that the small lateral approach made it possible to reduce the posterior facet accurately under direct vision and to obtain secure fixation that allowed early subtalar motion. Also, by using the medial approach, an accurate reduction of the tuberosity fragment relative to the superomedial fragment was possible. Paley and Hall [20] used only the medial approach for all his cases and reported that this approach was not adequate to address the lateral extrusion of bone fragment. This lateral extrusion lead to fibulocalcaneal impingement and irritation of the peroneal tendon sheath which is the most common cause of pain at the lateral aspect of hind foot. In the current study, small lateral approach was used for all cases on the premise that it was sufficient for the management of such fractures and to avoid the common wound problems encountered with the extensile approach especially in smokers and patients with diabetes. This approach was familiar, easy, simple, rapid, and adequate for the reduction and minimal fixation of the posterior facet; it also allowed for the insertion of bone graft and reduction of the lateral wall. However, it was difficult to visualize the far medial fracture of the posterior facet and to address the displaced medial wall fracture. The difficulty was overcome by closed manipulation of the tuberosity fragment in a way similar to that used by Essex-Lopresti [10].

McReynolds [18] and Stephenson [26] concluded that a medial approach is required more often for three-part tongue fractures. They explained the presence of a longer superomedial fragment in tongue fractures with more overriding medially. McReynolds [18], Burdeaux [5], and Stephenson [26] recommended the lateral approach to treat two-part tongue and joint depression fractures without much lateral displacement of the tuberosity fragment. In our opinion, a medial approach is more difficult; it carries a risk of neurovascular injury and the possibility of devascularization of the calcaneal fragments. It also provides less space for fixation of the tuberosity fragment to the superomedial fragment. However, it may be indicated in certain situations, e.g., when there is impalement of soft tissue preventing reduction of the medial wall.

Hammesfahr and Fleming [11], as well as Paley and Hall [20], reported better outcomes in patients who had a tongue type fracture than in those who had a joint depression fracture. They added that moderate comminution of the joint depression fractures worsened the prognosis, and extensively comminuted fractures were associated with the worst prognosis. We were not able to find a significant relation between the type of fracture and the functional results because of a small number of cases in this sample. However, $83.3 \%$ of patients with tongue-type fracture had satisfactory results, and $75 \%$ of patients with joint depression type had satisfactory results. We found also the more comminuted the fracture, the more unsatisfactory the results. Two patients with poor results had type III fractures, while majority of patients $(78.6 \%)$ with satisfactory results had type II fractures.

We agree with Leung et al. [15] that standard lateral, axial, and internal oblique radiographs are adequate for the assessment of the subtalar joint. Oblique radiographs of foot were valuable for addressing the extension of fracture into the calcaneocuboid joint. Our addition to the Sanders' classification was to allow greater definition of the extension of fractures anteriorly and the degree of comminution. Preoperative CT scan was useful for analysis of the fracture and planning; this helped to reduce surgical time and soft tissue morbidity. However, it is of less value in postoperative assessment because of interference by the metallic implants.

We found a strong correlation between the restoration of normal anatomy (congruity of the subtalar joint, Bohler's angle, calcaneal height and width, as assessed radiologically) and a satisfactory functional outcome. Stephenson [26] concluded that no patient who had a less than anatomical reduction of calcaneus had a good result. Leung et al. [15] found a significant correlation between the radiological assessment and the clinical findings with regard to the subtalar joint. Paley and Hall [20] stated that Bohler's angle is an indirect reflection of both calcaneal height and the arch angle; a small Bohler's angle is associated with a poor result. This implies that preservation of the calcaneal height and arch angle is important.

In McReynolds' series [18], in which the feet were immobilized in a plaster cast postoperatively, the motion of the subtalar joint at follow-up was $25 \%$ of normal in $90 \%$ of patients. In Stephenson's series [26], in which secure fixation was accomplished from the lateral side and early motion instituted, the average subtalar motion at follow-up was $75 \%$ of normal. In the present study in which motion was started early but guided by pain, the 
average subtalar motion at follow-up was $73.7 \%$ (ranges $45-98 \%$ ) of normal.

\section{Conclusions}

Despite the limitations of the current study, the single small lateral approach has proved to be simple, easy, safe, and adequate for management of displaced intra-articular calcaneal fractures. The medial approach is difficult, carries risk on injury to the neurovascular bundle, and may devascularize bone fragments. The minimal open reduction and internal fixation strategy described here can be a rewarding technique for closed displaced intra-articular calcaneal fractures with minimal comminution of the posterior facet and tuberosity fragment. For optimum results, restoration of near normal anatomy of the calcaneus and early institution of subtalar joint are needed.

\section{References}

1. Al-Mudhaffar M, Prassad CV, Mofidi A (2000) Wound complications following operative fixation of calcaneal fractures. Injury 31:461-464

2. Bezes H, Massart P, Delvaux D, Fourquet JP, Tzari F (1993) The operative treatment of intra-articular calcaneal fractures: indications, techniques, and results in 257 cases. Clin Orthop 290:55-59

3. Buckley R, Tough S, Mc Cormack R et al (2002) Operative compared with non operative treatment of displaced intra-articular calcaneal fractures. J Bone Joint Surg (Am) 84-A:1733-1744

4. Burdeaux BD Jr (1997) Fractures of the calcaneus: open reduction and internal fixation from the medial side a 21 -year prospective study. Foot Ankle 18:685-692

5. Burdeaux BD (1983) Reduction of calcaneal fractures by the McReynolds Medial Approach Technique and its experimental basis. Clin Orthop 177:87-103

6. Carr JB (1994) Surgical treatment of the intra-articular calcaneus fractures. Orthop North Am 25:665-675

7. Carr JB (2005) Surgical treatment of intra-articular calcaneal fractures: a review of small incision approach. J Orthop Trauma 19:109-117

8. Crosby LA, Fitzgibbons T (1990) Computerized tomography scanning of acute intra-articular fractures of the calcaneus: a new classification system. J Bone Joint Surg (Am) 72-A:852-859

9. Crosby LA, Fitzgibbons TC (1996) Open reduction and internal fixation of type II intra-articular calcaneus fractures. Foot Ankle $17: 253-258$

10. Essex-Lopresti P (1952) The mechanism, reduction technique, and results in fractures of the Os Calcis. Br J Surg 39:395-419
11. Hammesfahr R, Fleming LL (1981) Calcaneal fractures: a good prognosis. Foot Ankle 2:161-171

12. Harvey EJ, Grujic L, Early JS et al (2001) Morbidity associated with ORIF of intra-articular calcaneus fractures using a lateral approach. Foot Ankle Int 22:868-873

13. Knirk JL, Jupiter JB (1986) Intra-articular fractures of the distal end of the radius in young adults. J Bone Joint Surg (Am) 68-A:647-659

14. Leung KS, Chan WS, Shen WY et al (1989) Operative treatment of intra-articular fractures of the OsCalcis: the role of rigid internal fixation and primary bone grafting. J Orthop Trauma 3:232-240

15. Leung KS, Yuen KM, Chan WS (1993) Operative treatment of displaced intra-articular fractures of the calcaneum: medium-term results. J Bone Joint Surg 75-B:196-201

16. Marcey LR, Benirschke SK, Sangeorzan BJ, Hansen ST Jr (1994) Acute calcaneal fractures: treatment options and results. J Am Acad Orthop Surg 2:36-43

17. McMaster M (1976) Disability of the hindfoot after fracture of the tibial shaft. J Bone Joint Surg (Br) 58-B:90-93

18. McReynold IS (1982) Trauma to the Os calcis and heel cord. In: Jahss MH (ed) Disorders of the foot. WB Saunders, Philadelphia, pp 1497-1542

19. Murphy GA (1998) Fracture and dislocations of foot. In: Canale ST (ed) Campbell's operative orthopaedics. Mosby-Year Book, St Louis-Missouri, pp 1924-1971

20. Paley D, Hall H (1993) Intra-articular fractures of the calcaneus: a critical analysis of results and prognostic factors. J Bone Joint Surg (A) 75-A:342-354

21. Perry J (1983) Anatomy and biomechanics of the hindfoot. Clin Orthop 177:9-15

22. Pozo JL, Kirwan EOG, Jackson AM (1984) The long-term results of conservative management of severely displaced fractures of the calcaneus. J Bone Joint Surg (Br) 66-B:386-390

23. Sanders R, Fortin P, DiPasquate T, Walling A (1993) Operative treatment in 120 displaced intra-articular calcaneal fractures: results using a prognostic computed tomography scan classification. Clin Orthop Relat Res 290:87-95

24. Sarrafian SK (1983) Anatomy of the foot and ankle. JB lippincott, Philadelphia, p 157

25. Stephenson JR (1983) Displaced fractures of the Os Calcis involving the subtalar joint. Key Ankle 4:91-101

26. Stephenson JR (1987) Treatment of displaced intra-articular fractures of the calcaneus using medial and lateral approaches, internal fixation, and early motion. J Bone Joint Surg (Am) 69-A:115-130

27. Thordarson DB, Krieger LE (1996) Operative Vs. Nonoperative treatment of intra-articular fractures of the calcaneus: a prospective randomized trial. Foot Ankle 17:2-9

28. Thordarson DB, Latterier M (2003) Open reduction and internal fixation of calcaneal fractures with a low profile titanium perimeter plate. Foot Ankle Int 24:217-221

29. Wei SY, Okere E, Esmail AN, Bor CT, Delong WG (2001) Operatively treated calcaneus fractures: to mobilize or not to mobilize. Univ Penn Orthop J 14:71-73 